\title{
ANÁLISE DE ERROS ORTOGRÁFICOS EM DIFERENTES PROBLEMAS DE APRENDIZAGEM
}

\author{
Analyzing typical orthographic mistakes related \\ to different learning problems
}

Jaime Luiz Zorzi ${ }^{(1)}$, Sylvia Maria Ciasca ${ }^{(2)}$

\begin{abstract}
RESUMO
Objetivos: descrever achados ortográficos em problemas de aprendizagem, verificar se os erros produzidos são aqueles encontrados na aprendizagem normal e analisar se predominam problemas de natureza ortográfica ou fonológica. Métodos: examinou-se a escrita de 64 sujeitos avaliados pelo Laboratório de Distúrbios de Aprendizagem do Departamento de Neurologia da UNICAMP, diagnosticados com algum problema de aprendizagem: Transtorno do Déficit de Atenção/Hiperatividade (28); Dificuldades de Aprendizagem (13); Distúrbio de Aprendizagem (7); Dislexia (3); Distúrbios Associados (5) e Diagnóstico inconclusivo (9). As idades variaram entre 8;2 e 13;4 anos, média 10;6 anos. Foram incluídos sujeitos alfabetizados, sem rebaixamento intelectual. Os erros foram classificados em 11 categorias e quantificados para análise estatística. Resultados: os erros correspondem àqueles observados em crianças sem queixa de aprendizagem. Os erros por Representações Múltiplas, Omissão de Letras e Oralidade, são respectivamente, os três tipos mais frequentes nos casos de Transtorno do Déficit de Atenção e Hiperatividade, Dificuldades Escolares, Distúrbios Associados e Diagnóstico Desconhecido. No Distúrbio de Aprendizagem a sequência é de Representações Múltiplas, Omissão, Outras Alterações e Surdas-sonoras. Na dislexia observa-se a sequência de Representações Múltiplas, Oralidade, Omissão e Outras Alterações. Existe uma tendência geral de predomínio das alterações ortográficas, embora sem diferença significante em relação aos erros de natureza fonológica. Conclusão: os erros de natureza ortográfica são os mais frequentes em relação aos de natureza fonológica. Com tendência contrária, os erros visuo-espaciais têm baixa ocorrência em geral, o que mostra que a dificuldade de todos os grupos é fundamentalmente de origem linguística e não perceptual.
\end{abstract}

DESCRITORES: Transtornos de Aprendizagem; Escrita Manual; Educação

\section{INTRODUÇÃO}

O desenvolvimento de habilidades básicas para ler e escrever, graças ao seu impacto na educação recebe uma atenção especial, principalmente nas séries iniciais do ensino fundamental. Entretanto, tal aprendizagem pode ser um grande desafio para muitos e dificuldades variáveis podem surgir durante este processo. Neste sentido, um dos aspectos que

(1) Fonoaudiólogo; Professor do CEFAC - Pós-Graduação em Saúde e Educação; Doutor em Educação pela Universidade Estadual de Campinas.

(2) Psicóloga; Professora da Universidade Estadual de Campinas, UNICAMP, Campinas, SP; Doutora em Neurociências pela Universidade Estadual de Campinas.

Conflito de Interesses: Inexistente chama a atenção, diz respeito à ortografia, isto é, ao domínio da escrita convencional das palavras. Pode ser difícil, para muitas crianças, compreender como as palavras devem ser apropriadamente grafadas, o que pode ser observado nas alterações ortográficas presentes em suas produções escritas.

Tais fatos têm merecido a atenção de muitos pesquisadores, gerando estudos comparativos sobre a complexidade ortográfica de diferentes línguas, o que pode torná-las mais ou menos transparentes e assim influenciar sua aprendizagem, facilitando-a ou dificultando-a ${ }^{1-4}$. Têm sido apontadas escritas com ortografias profundas e escritas com ortografias mais superficiais. $\mathrm{O}$ aspecto fundamental das diferenças reside na complexidade silábica e ortográfica. Na leitura, as estruturas silábicas 
afetariam principalmente a decodificação enquanto que a profundidade ortográfica afetaria a leitura tanto de palavras reais quanto de pseudopalavras ${ }^{5}$.

Uma atenção maior também tem sido dada às alterações ortográficas do português escrito no Brasil, com a finalidade de melhor compreendê-las ${ }^{6-14}$. Embora variem em termos de análises mais quantitativas ou qualitativas, de uma maneira uniforme, estes estudos indicam que os "erros" fazem parte da aprendizagem, podendo revelar hipóteses de que as crianças vão, gradativamente, construindo para chegar a conhecimentos mais aprofundados sobre a escrita.

Desta forma, observa-se que as crianças cometem "erros" durante a aprendizagem da escrita até que, progressivamente, elas dominem de forma mais segura o sistema ortográfico. Consequentemente, os erros tornam-se cada vez mais específicos e ocasionais. Porém, por outro lado, também se observa que algumas delas parecem ter uma trajetória diferente, exibindo uma diversidade e frequência de alterações de escrita mais intensa e duradoura. Tais dificuldades podem, além de revelarem uma possível má qualidade de ensino, ser sintomas de problemas ou limitações, como os distúrbios de aprendizagem e as dislexias 1,3,12,13,15-21. Muitas destas crianças acabam sendo encaminhadas para profissionais especializados para diagnóstico e atendimento extra-escolar.

A leitura e a escrita envolvem o uso de estratégias fonológicas e ortográficas, sendo que algumas crianças podem ter grandes dificuldades com os aspectos fonológicos enquanto que outras podem ter tais limitações quanto aos aspectos ortográficos. Poderia haver uma influência genética mais forte sobre as dificuldades fonológicas, enquanto que os déficits de natureza ortográfica teriam maior influência ambiental, dado seu caráter convencional ${ }^{22}$. Têm sido apontadas dificuldades na representação da estrutura fonológica em palavras mesmo em disléxicos aprendendo a ortografia de escritas consideras como mais transparentes ${ }^{1}$. Neste sentido, a dislexia do desenvolvimento estaria mais relacionada aos déficits de decodificação de origem fonológica. Por sua vez, os déficits ortográficos estariam ligados a um atraso mais geral do desenvolvimento da linguagem, determinado por insuficiente exposição da criança à linguagem escrita ${ }^{22}$.

A existência de aspectos fonológicos e ortográficos determinando a escrita das palavras pode levar a diversos tipos de erros os quais também podem ter frequências distintas de ocorrência. Esta incidência variável reforça a hipótese de que a presença destes desvios pode ser influenciada por características intrínsecas da própria linguagem escrita, as quais demandam diferentes habilidades ou conhecimentos por parte do aprendiz ${ }^{6,12,18-21,23-27} . \mathrm{Na}$ ortografia do inglês, por exemplo, fatores como frequência e comprimento das palavras, assim como os modos de combinação das letras têm sido apontados como aspectos associados às dificuldades na soletração e na leitura, ao ponto de levar autores ao questionamento se não seria o inglês escrito, em si, uma língua disléxica ${ }^{24}$.

A aprendizagem depende de condições estruturais e funcionais do sistema nervoso central. Disfunções que ocorram nas etapas de desenvolvimento das unidades funcionais cerebrais podem acarretar alterações na linguagem e na aprendizagem. Problemas de atenção e concentração são derivados de disfunções nas zonas primárias. Dificuldades na aprendizagem envolvendo a leitura, a escrita e a matemática podem ser derivadas de disfunções nas zonas secundárias. Por sua vez, o baixo rendimento cognitivo, falhas na compreensão da linguagem falada, limitações em termos de leitura escrita e matemática, estão relacionadas a disfunções nas áreas terciárias ${ }^{28}$.

Mais especificamente, existem evidências de que os diferentes tipos de dislexia podem estar associados a diferentes disfunções de áreas corticais específicas: a dislexia fonológica resultaria de disfunções no lobo temporal; a dislexia diseidética estaria associada a disfunções do lobo occipital e a dislexia mista a alterações funcionais do lobo préfrontal, frontal, occipital e temporal ${ }^{28}$. Tais achados têm sido alvo de muitas investigações graças aos avanços dos exames por meio de neuroimagens, particularmente as imagens obtidas através da ressonância magnética funcional, a fim de se estudar a ativação cerebral durante o processamento de linguagem, em condições variadas de apresentação de estímulos. Diferenças no funcionamento cerebral, nas áreas envolvidas no processamento de linguagem de crianças apresentando problemas de leitura e escrita têm sido confirmadas ${ }^{29}$.

Deve-se considerar que a apropriação do sistema de escrita é um processo evolutivo no qual o aprendiz elabora hipóteses ou ideias a respeito do que é a escrita, as quais revelam diferentes graus de conhecimentos que estão sendo constituídos. Isto significa que não se aprende a escrever de imediato e que "erros" estão implícitos em tal processo ${ }^{6}$.

Porém, a permanência de erros pode refletir déficits que produzem dificuldades ou lentidão acentuada no sentido de a criança gerar e generalizar hipóteses que, sucessivamente, permitam a apreensão cada vez mais aprofundada da escrita. Tais crianças estabilizariam hipóteses elementares e insuficientes a respeito da ortografia. Uma série de conhecimentos apontados como fundamentais, 
principalmente a precisão das representações fonológicas e o conhecimento das convenções ortográficas, não estariam sendo adequadamente desenvolvidos, provocando as dificuldades na escrita das palavras ${ }^{10,11,30,31}$.

Os diversos estudos sobre dificuldades em leitura, assim como em soletração e ortografia têm sido feitos principalmente com crianças disléxicas ${ }^{1,2,4,17,23,32,33}$. Por outro lado, pouca atenção tem sido dada a outros tipos de problemas, como os distúrbios específicos de linguagem ${ }^{18,34}$. Pode-se também apontar a escassez de pesquisas envolvendo os problemas ortográficos nos distúrbios de aprendizagem, déficits de atenção e outros transtornos que comumente são encaminhados para diagnóstico e intervenção.

Uma das questões que merecem investigação mais aprofundada diz respeito à análise dos tipos de erros mais comumente encontrados nas crianças apresentando transtornos de aprendizagem. Seriam eles de natureza diversa daqueles que as crianças sem dificuldades apresentam, ou seriam semelhantes, porém resistindo por mais tempo?

Outra questão, pouco explorada, é verificar, considerando-se os vários tipos distintos de problemas que compõem os transtornos de aprendizagem, se haveria diferenças entre eles, ou seja, se os erros ortográficos produzidos por disléxicos, por exemplo, seriam diferentes ou semelhantes àqueles encontrados nos distúrbios de aprendizagem e outros grupos. A mesma pergunta pode ser feita em relação à frequência de ocorrências dos erros: haveria ou não diferenças entre os diversos grupos?

Considerando-se que os erros ortográficos estão presentes em muitas crianças apresentando diversos tipos de problemas de aprendizagem, e não unicamente na dislexia, o objetivo desta pesquisa é o de analisar a produção escrita de crianças classificadas nestas condições. Mais especificamente, a partir de escritos de grupos de crianças diagnosticadas como tendo alguma limitação em termos de aprendizado, serão quantificados e classificados os erros encontrados tendo em vista identificar o perfil ortográfico de cada grupo de problema. Com este estudo pretende-se apontar possíveis perfis ortográficos que possam ser indicadores das alterações de escrita que caracterizam os diversos transtornos de aprendizagem. Os seguintes objetivos específicos dirigem este trabalho:

- Traçar o perfil ortográfico de escolares com diversos tipos de problemas de aprendizagem;

- Verificar se os tipos de erros produzidos são os mesmos encontrados na aprendizagem considerada normal;
- Verificar se predominam problemas de natureza ortográfica ou de natureza fonética nos diferentes tipos de problemas.

\section{MÉTODOS}

Faz parte desta pesquisa um grupo de 64 sujeitos que passaram pelo processo avaliativo do Laboratório de Distúrbios de Aprendizagem do Departamento de Neurologia da Faculdade de Ciências Médicas da UNICAMP (DISAPRE) e que foram diagnosticados como apresentando algum tipo de problema de aprendizagem. As idades variaram entre oito anos e dois meses e treze anos e quatro meses, com uma média de dez anos e seis meses. Tais sujeitos, de ambos os sexos, que frequentam desde a primeira série do ensino fundamental até o segundo ano do ensino médio de escolas públicas e particulares, chegam com a queixa de problemas na aprendizagem para serem avaliados pela equipe multiprofissional do Laboratório, tendo em vista o diagnóstico diferencial e encaminhamentos: avaliação neurológica, neuropsicológica e fonoaudiológica. Foram incluídos somente sujeitos que já haviam alcançado a fase alfabética de aquisição da escrita e que não apresentavam qualquer tipo de rebaixamento intelectual. A amostra ficou caracterizada da seguinte maneira: Transtorno do Déficit de Atenção/Hiperatividade (TDA/H - 28); Dificuldades de Aprendizagem (13); Distúrbio de Aprendizagem (7); Dislexia (3); Distúrbios Associados (5) e Diagnóstico inconclusivo (9).

O procedimento para coleta do material escrito está composto de duas partes: um ditado de palavras reais e inventadas, previamente elaborado para medir uma série de conhecimentos ortográficos, e de uma redação a partir de um tema dado ("Uma casa abandonada"), por meio da qual é verificado o nível de domínio da ortografia em situação de produção de escrita espontânea. O material escrito foi coletado durante o processo de avaliação/diagnóstico por um avaliador previamente orientado quanto à forma de propor a situação para obtenção do material. Os erros foram analisados de acordo com os critérios de Zorzi ${ }^{6}$.

Compõem a pesquisa os sujeitos cujos responsáveis permitiram, por escrito, sua participação a partir dos esclarecimentos contidos no Termo de Consentimento Livre e Esclarecido. Este projeto foi aprovado no Comitê de Ética e Pesquisa do CEFAC - Pós-Graduação em Saúde e Educação, tendo sido aprovado e registrado sob o no 025/08.

O tratamento estatístico foi realizado a partir do emprego dos seguintes testes: Wilcoxon, Igualdade de Duas Proporções, Correlação de Spearman, Teste de Correlação, Intervalo de Confiança para Média e P-valor. O nível de significância adotado para este trabalho é o de 0,05 (5\%). 


\section{RESULTADOS}

O grupo diagnosticado como Transtorno do Déficit de Atenção e Hiperatividade $(T D A / H)$ é o mais numeroso, denotando uma diferença estatisticamente significante em relação a todos os demais. De acordo com a Tabela 1, existe diferença entre os tipos de erros do Grupo TDA/H de modo geral. Para uma análise mais detalhada, a Tabela 2 compara todos os erros, aos pares, exibindo os p-valores de tais comparações duas a duas (Teste de Wilcoxon) entre os erros. O erro por "Representações Múltiplas" é o tipo de maior resultado e considerado estatisticamente diferente dos demais $(p<0,001)$. Os erros por "Omissão" e "Apoio na Oralidade" aparecem em segundo e terceiro lugares, sem diferença estatisticamente significante entre eles, porém com diferença significante em relação aos demais erros. "Outras alterações" aparecem em quarto lugar, mas não apresentando diferença estatisticamente significante em relação aos tipos de erros seguintes, com os quais forma uma espécie de bloco: "Junção-separação", "Trocas surdas/ sonoras", "Am x ão". "Acréscimo de letras" é o oitavo tipo de erro mais frequente, sem diferença significante em relação à "Generalização" mas que, apesar da baixa ocorrência, apresenta diferença significante quando comparada às "Inversões" e "Letras Parecidas". Estes dois últimos erros, os menos frequentes, também não apresentam diferenças entre si.

A Tabela 3 mostra que existe diferença estatística entre os diversos tipos de erros. O erro com maior resultado foi "Representações Múltiplas", com média de $15,85( \pm 5,14)$, mas que segundo a Tabela 4 , de p-valores, não pode ser considerado estatisticamente diferente do erro "Omissão", com média $14,92( \pm 9,82)$. Em seguida vem o erro por "Apoio na Oralidade", com diferença significante com relação a todos os demais, com exceção de "Omissão". Sem diferença significante entre os mesmos, vem

Tabela 1 - Descritiva dos erros para o problema TDA/H

\begin{tabular}{lccccccccc}
\hline TDA/H & Média & Mediana & $\begin{array}{c}\text { Desvio } \\
\text { Padrão }\end{array}$ & CV & Q1 & Q3 & N & IC & p-valor \\
\hline $\begin{array}{l}\text { representações } \\
\text { múltiplas }\end{array}$ & 18,85 & 19 & 7,20 & $38 \%$ & 13,5 & 23,5 & 27 & 2,72 & \\
oralidade & 5,63 & 5 & 3,83 & $68 \%$ & 3 & 7,5 & 27 & 1,45 & \\
omissão & 7,58 & 4,5 & 9,02 & $119 \%$ & 2,25 & 9,5 & 26 & 3,47 & \\
junção/separação & 2,78 & 1 & 3,47 & $125 \%$ & 0,5 & 3,5 & 27 & 1,31 & \\
am / ão & 1,85 & 1 & 2,74 & $148 \%$ & 0 & 2 & 27 & 1,03 & $<0,001$ \\
generalização & 1,22 & 1 & 1,50 & $123 \%$ & 0 & 2 & 27 & 0,57 & $*$ \\
surdas/sonoras & 2,07 & 1 & 3,44 & $166 \%$ & 0 & 2,5 & 27 & 1,30 & \\
acréscimo & 1,33 & 0 & 1,92 & $144 \%$ & 0 & 2 & 27 & 0,72 & \\
letras parecidas & 0,56 & 0 & 1,15 & $208 \%$ & 0 & 0,5 & 27 & 0,44 & \\
inversões & 0,59 & 0 & 1,95 & $328 \%$ & 0 & 0 & 27 & 0,73 & \\
outras & 3,63 & 2 & 5,98 & $165 \%$ & 0 & 4 & 27 & 2,26 & \\
\hline
\end{tabular}

Tabela 2 - P-valores das comparações entre tipos de erros em TDA/H (sim)

\begin{tabular}{|c|c|c|c|c|c|c|c|c|c|c|}
\hline TDA/H & $\begin{array}{l}\text { representações } \\
\text { múltiplas }\end{array}$ & oralidade & omissão & junção/sep. & am / ão & general & sur/son. & acréscimo & letras par. & inversões \\
\hline oralidade & $<0,001$ & & & & & & & & & \\
\hline omissão & $<0,001$ & 0,760 & & & & & & & & \\
\hline junção/sep. & $<0,001$ & 0,008 & 0,001 & & & & & & & \\
\hline am / ão & $<0,001$ & 0,001 & 0,002 & 0,240 & & & & & & \\
\hline general. & $<0,001$ & $<0,001$ & $<0,001$ & 0,052 & 0,504 & & & & & \\
\hline sur/son. & $<0,001$ & 0,001 & 0,002 & 0,374 & 0,958 & 0,482 & & & & \\
\hline acréscimo & $<0,001$ & $<0,001$ & $<0,001$ & 0,117 & 0,734 & 0,692 & 0,713 & & & \\
\hline letras par. & $<0,001$ & $<0,001$ & $<0,001$ & 0,001 & 0,016 & 0,064 & 0,031 & 0,015 & & \\
\hline inversões & $<0,001$ & $<0,001$ & $<0,001$ & 0,001 & 0,023 & 0,015 & 0,032 & 0,032 & 0,863 & \\
\hline outras & $<0,001$ & 0,020 & 0,004 & 0,648 & 0,166 & 0,099 & 0,123 & 0,018 & 0,001 & $<0,001$ \\
\hline
\end{tabular}


Tabela 3 - Descritiva dos erros para o problema Dificuldade Escolar (sim)

\begin{tabular}{lccccccccc}
\hline Dificuldade escolar & Média & Mediana & $\begin{array}{c}\text { Desvio } \\
\text { Padrão }\end{array}$ & CV & Q1 & Q3 & N & IC & p-valor \\
\hline representações & 15,85 & 15 & 9,45 & $60 \%$ & 8 & 21 & 13 & 5,14 & \\
múltiplas & 8,62 & 6 & 7,25 & $84 \%$ & 3 & 14 & 13 & 3,94 & \\
oralidade & 14,92 & 5 & 18,06 & $121 \%$ & 2 & 31 & 13 & 9,82 & \\
omissão & 3,08 & 2 & 2,75 & $89 \%$ & 1 & 5 & 13 & 1,50 & \\
junção/separação & 1,38 & 1 & 2,02 & $146 \%$ & 0 & 1 & 13 & 1,10 & $<0,001$ \\
am / ão & 0,85 & 0 & 1,14 & $135 \%$ & 0 & 1 & 13 & 0,62 & $\star$ \\
generalização & 2,77 & 1 & 3,32 & $120 \%$ & 0 & 4 & 13 & 1,81 & \\
Surda/sonora & 1,92 & 1 & 2,50 & $130 \%$ & 0 & 2 & 13 & 1,36 & \\
acréscimo & 0,54 & 0 & 0,97 & $180 \%$ & 0 & 1 & 13 & 0,53 & \\
letras parecidas & 0,31 & 0 & 1,11 & $361 \%$ & 0 & 0 & 13 & 0,60 & \\
inversões & 4,77 & 1 & 7,18 & $151 \%$ & 0 & 5 & 13 & 3,90 & \\
outras & & & & & & & & &
\end{tabular}

Tabela 4 - P-valores das comparações entre tipos de erros em Dificuldade Escolar (sim)

\begin{tabular}{|c|c|c|c|c|c|c|c|c|c|c|}
\hline $\begin{array}{l}\text { Dificuldade } \\
\text { escolar }\end{array}$ & $\begin{array}{c}\text { representações } \\
\text { múltiplas }\end{array}$ & oralidade & omissão & junção/sep. & am / ão & general. & sur/son. & acréscimo & letras par. & inversões \\
\hline oralidade & 0,002 & & & & & & & & & \\
\hline omissão & 0,754 & 0,238 & & & & & & & & \\
\hline junção/sep. & 0,001 & 0,015 & 0,014 & & & & & & & \\
\hline am / ão & 0,001 & 0,005 & 0,005 & 0,015 & & & & & & \\
\hline general. & 0,001 & 0,003 & 0,005 & 0,040 & 0,375 & & & & & \\
\hline sur/son. & 0,001 & 0,013 & 0,023 & 0,669 & 0,209 & 0,114 & & & & \\
\hline acréscimo & 0,001 & 0,002 & 0,003 & 0,177 & 0,765 & 0,280 & 0,472 & & & \\
\hline letras par. & 0,001 & 0,002 & 0,002 & 0,010 & 0,167 & 0,429 & 0,024 & 0,072 & & \\
\hline inversões & 0,001 & 0,002 & 0,002 & 0,003 & 0,105 & 0,233 & 0,028 & 0,011 & 0,581 & \\
\hline outras & 0,002 & 0,026 & 0,010 & 0,944 & 0,233 & 0,103 & 0,753 & 0,057 & 0,041 & 0,012 \\
\hline
\end{tabular}

o bloco formado por "Outras alterações", "Junção/ separação", "Surdas/sonoras", "Acréscimo", "Am x ão" e "Generalização". As médias mais baixas ficam novamente com "Letras parecidas e inversões".

A Tabela 5 demonstra que existem diferenças estatisticamente significantes entre diversos erros para o grupo "Distúrbio de aprendizagem". O erro com maior resultado é "Representações Múltiplas", com média de $15,43( \pm 9,08)$. A Tabela 6 , de $p$-valores, indica que não há diferença estatisticamente significante entre este erro de maior ocorrência em relação a "Omissão" $(9,71 \pm 13,10)$ e "Outras alterações" $(6,71 \pm 8,53)$. Ainda na ordem de frequência, os erros "Surdas/sonoras" e "Oralidade" parecem formar um segundo grupo, mas que, embora tenham uma média mais elevada, não apresentam uma diferença estatisticamente significante em relação aos demais tipos de erros que se seguem, com exceção das inversões $(p=0,042)$.

$\mathrm{Na}$ Tabela 7, embora existam diferenças entre os erros, não se pode afirmar se as mesmas são significantes ou não uma vez que a amostra deste grupo se limita a 3 sujeitos. Observa-se um primeiro conjunto de erros formado por "Representações Múltiplas", "Apoio na Oralidade" e "Omissões". Um segundo bloco está constituído por "Outras alterações", "Surdas/sonoras" e "Am x ao". O conjunto de erros menos observado segue a ordem "Junção / separação", "Acréscimo", "Generalização", "Inversões" e "Letras Parecidas".

De acordo com a Tabela 8, existe diferença estatística entre os erros para este tipo de problema. O erro com maior resultado foi "Representações Múltiplas" com média de $18( \pm 6,44)$, mas que segundo a Tabela 9 não pode ser considerado estatisticamente diferente do erro tipo "Omissão", com média de $11,83( \pm 8,61)$. Um segundo agrupamento de erros, sem diferença estatística significante entre eles é composto por "Oralidade", "Outras alterações", "Surdas/sonoras", "Am x ão" e "Acréscimo". O último conjunto de erros é constituído por "Junção/separação", "Letras Parecidas", "Inversões" e "Generalização", sem que haja diferença estatística entre tais tipos de erros. 
Tabela 5 - Descritiva dos erros para o problema Distúrbio de Aprendizagem (sim)

\begin{tabular}{lccccccccc}
\hline $\begin{array}{l}\text { Distúrbio de } \\
\text { aprendizagem }\end{array}$ & Média & Mediana & $\begin{array}{l}\text { Desvio } \\
\text { Padrão }\end{array}$ & CV & Q1 & Q3 & N & IC & p-valor \\
\hline $\begin{array}{l}\text { representações } \\
\text { múltiplas }\end{array}$ & 15,43 & 11 & 12,26 & $79 \%$ & 8,5 & 22,5 & 7 & 9,08 & \\
oralidade & 6,29 & 5 & 4,64 & $74 \%$ & 3,5 & 10 & 7 & 3,44 & \\
omissão & 9,71 & 2 & 17,69 & $182 \%$ & 0,5 & 8,5 & 7 & 13,10 & \\
junção/separação & 2,57 & 3 & 1,99 & $77 \%$ & 1 & 4 & 7 & 1,47 & \\
am / ão & 2,43 & 3 & 1,72 & $71 \%$ & 1,5 & 3,5 & 7 & 1,27 & \\
generalização & 1,14 & 1 & 0,69 & $60 \%$ & 1 & 1,5 & 7 & 0,51 & $0,004^{*}$ \\
surda/sonora & 7,00 & 1 & 10,80 & $154 \%$ & 0,5 & 10,5 & 7 & 8,00 & \\
acréscimo & 1,29 & 1 & 1,80 & $140 \%$ & 0 & 1,5 & 7 & 1,33 & \\
letras parecidas & 2,43 & 0 & 5,56 & $229 \%$ & 0 & 1 & 7 & 4,12 & \\
inversões & 1,14 & 0 & 2,04 & $178 \%$ & 0 & 1,5 & 7 & 1,51 & \\
outras & 6,71 & 1 & 11,51 & $171 \%$ & 0,5 & 7 & 7 & 8,53 & \\
\hline
\end{tabular}

Tabela 6 - P-valores das comparações entre tipos de erros em Distúrbio de Aprendizagem (sim)

\begin{tabular}{|c|c|c|c|c|c|c|c|c|c|c|}
\hline $\begin{array}{l}\text { Distúrbio de } \\
\text { aprendizagem }\end{array}$ & $\begin{array}{c}\text { representações } \\
\text { múltiplas }\end{array}$ & oralidade & omissão & junção/sep. & am / ão & general. & sur/son. & acréscimo & letras par. & inversões \\
\hline oralidade & 0,027 & & & & & & & & & \\
\hline omissão & 0,310 & 0,733 & & & & & & & & \\
\hline junção/sep. & 0,027 & 0,078 & 0,735 & & & & & & & \\
\hline am / ão & 0,028 & 0,058 & 0,674 & 0,786 & & & & & & \\
\hline general. & 0,028 & 0,042 & 0,263 & 0,056 & 0,056 & & & & & \\
\hline sur/son. & 0,044 & 1,000 & 0,750 & 0,684 & 0,674 & 0,336 & & & & \\
\hline acréscimo & 0,027 & 0,027 & 0,168 & 0,077 & 0,168 & 1,000 & 0,180 & & & \\
\hline letras par. & 0,028 & 0,075 & 0,066 & 0,462 & 0,480 & 0,492 & 0,072 & 0,596 & & \\
\hline inversões & 0,028 & 0,027 & 0,042 & 0,176 & 0,136 & 0,666 & 0,042 & 0,496 & 0,593 & \\
\hline outras & 0,173 & 0,715 & 0,201 & 0,752 & 0,673 & 0,285 & 0,892 & 0,223 & 0,465 & 0,042 \\
\hline
\end{tabular}

Tabela 7 - Descritiva dos erros para o problema Dislexia

\begin{tabular}{lccccccccc}
\hline Dislexia & Média & Mediana & $\begin{array}{c}\text { Desvio } \\
\text { Padrão }\end{array}$ & CV & Q1 & Q3 & N & IC & $\begin{array}{c}\text { p- } \\
\text { valor }\end{array}$ \\
\hline representações & 27,67 & 26 & 5,69 & $21 \%$ & 24,5 & 30 & 3 & 6,43 & \\
múltiplas & 20,33 & 20 & 4,51 & $22 \%$ & 18 & 22,5 & 3 & 5,10 & \\
oralidade & 18,00 & 10 & 17,44 & $97 \%$ & 8 & 24 & 3 & 19,73 & \\
omissão & 3,33 & 3 & 1,53 & $46 \%$ & 2,5 & 4 & 3 & 1,73 & \\
junção/separação & 8,67 & 3 & 10,69 & $123 \%$ & 2,5 & 12 & 3 & 12,10 & \\
am / ão & 2,33 & 3 & 2,08 & $89 \%$ & 1,5 & 3,5 & 3 & 2,36 & $0,057 \#$ \\
generalização & 10,00 & 2 & 13,86 & $139 \%$ & 2 & 14 & 3 & 15,68 & \\
Surda/sonora & 2,67 & 2 & 2,08 & $78 \%$ & 1,5 & 3,5 & 3 & 2,36 & \\
acréscimo & 1,33 & 1 & 1,53 & $115 \%$ & 0,5 & 2 & 3 & 1,73 & \\
letras parecidas & 1,67 & 1 & 2,08 & $125 \%$ & 0,5 & 2,5 & 3 & 2,36 & \\
inversões & 11,67 & 12 & 8,50 & $73 \%$ & 7,5 & 16 & 3 & 9,62 & \\
outras & & & & & & & &
\end{tabular}


Tabela 8 - Descritiva dos erros para o problema Associados

\begin{tabular}{lccccccccc}
\hline Associados & Média & Mediana & $\begin{array}{c}\text { Desvio } \\
\text { Padrão }\end{array}$ & CV & Q1 & Q3 & N & IC & p-valor \\
\hline representações & 18,00 & 16,5 & 8,05 & $45 \%$ & 13,8 & 21,5 & 6 & 6,44 & \\
múltiplas & 7,83 & 6 & 6,24 & $80 \%$ & 4,25 & 7,75 & 6 & 4,99 & \\
oralidade & 11,83 & 8 & 10,76 & $91 \%$ & 5,25 & 19,8 & 6 & 8,61 & \\
omissão & 1,33 & 1 & 1,51 & $113 \%$ & 0 & 2,75 & 6 & 1,20 & \\
junção/separação & 5,00 & 2,5 & 8,00 & $160 \%$ & 0,5 & 3,75 & 6 & 6,40 & \\
am / ão & 0,67 & 0,5 & 0,82 & $122 \%$ & 0 & 1 & 6 & 0,65 & $<0,001^{*}$ \\
generalização & 5,83 & 2 & 10,13 & $174 \%$ & 0 & 4,75 & 6 & 8,10 & \\
Surda/sonora & 2,67 & 2,5 & 2,25 & $84 \%$ & 1 & 4,75 & 6 & 1,80 & \\
acréscimo & 1,00 & 0 & 1,67 & $167 \%$ & 0 & 1,5 & 6 & 1,34 & \\
letras parecidas & 0,83 & 0,5 & 0,98 & $118 \%$ & 0 & 1,75 & 6 & 0,79 & \\
inversões & 6,00 & 3,5 & 6,63 & $111 \%$ & 1,5 & 9,25 & 6 & 5,31 & \\
outras & & & & & & & & & \\
\hline
\end{tabular}

Tabela 9 - P-valores das comparações entre tipos de erros em Associados

\begin{tabular}{|c|c|c|c|c|c|c|c|c|c|c|}
\hline Associados & $\begin{array}{c}\text { representações } \\
\text { múltiplas }\end{array}$ & oralidade & omissão & junção/sep. & am / ão & general. & sur/son. & acréscimo & letras par. & inversões \\
\hline oralidade & 0,028 & & & & & & & & & \\
\hline omissão & 0,248 & 0,462 & & & & & & & & \\
\hline junção/separação & 0,028 & 0,043 & 0,043 & & & & & & & \\
\hline am / ão & 0,027 & 0,078 & 0,225 & 0,279 & & & & & & \\
\hline generalização & 0,028 & 0,026 & 0,046 & 0,336 & 0,141 & & & & & \\
\hline surda/sonora & 0,046 & 0,343 & 0,225 & 0,223 & 0,465 & 0,223 & & & & \\
\hline acréscimo & 0,026 & 0,043 & 0,042 & 0,223 & 0,785 & 0,104 & 1,000 & & & \\
\hline letras parecidas & 0,028 & 0,043 & 0,043 & 0,581 & 0,109 & 0,581 & 0,273 & 0,223 & & \\
\hline inversões & 0,028 & 0,027 & 0,043 & 0,496 & 0,109 & 0,705 & 0,109 & 0,042 & 1,000 & \\
\hline outras & 0,043 & 0,686 & 0,043 & 0,223 & 0,715 & 0,058 & 0,500 & 0,144 & 0,068 & 0,043 \\
\hline
\end{tabular}

Considerando-se a Tabela 10, existe diferença estatística entre os erros para este tipo de problema $(p<0,001)$. Na Tabela 11, de $p$-valores, o erro com maior resultado, que é "Representações Múltiplas", com média de 15,25 $( \pm 5,85)$, não pode ser considerado estatisticamente diferente do erro tipo "Omissão", com média de 10,75 $( \pm 9,33)$. Na sequência, um segundo grupo de erros é formado por "Oralidade e outras", sem diferença estatística entre os mesmos. Um terceiro bloco é composto por "Acréscimos", "Surdas/sonoras", "Am x ão", "Generalização", "Junção/Separação" e "Letras parecidas", também sem diferenças significantes entre eles. $O$ erro "Inversões" fica na última posição.

\section{DISCUSSÃO}

Os erros encontrados nos escritos dos sujeitos desta pesquisa correspondem àqueles também encontrados em crianças sem queixa de aprendizagem da escrita ${ }^{6}$. Não se observa uma categoria distinta ou específica, mas sim possíveis variações em termos de frequência de ocorrência.

As categorias de erros usadas neste trabalho podem ser divididas em dois grupos, de acordo com sua natureza predominantemente fonológica ou ortográfica. As "Representações Múltiplas", a "Oralidade", "Junção/separação", "Am/ão" e "Generalização", correspondem a erros de substituição de uma letra por outra por desconhecimento ou desconsideração de aspectos envolvendo regras ortográficas, sendo que a análise fonológica da palavra a ser escrita ocorreu de modo adequado. Quando uma criança escreve "cabeça" com a letra "s" ("cabesa") os aspectos fonológicos estão adequados porque a letra "s" pode escrever o som /s/. Porém, em razão de uma regra contextual, a letra "s", nesta posição fica com o som /z/.

Os erros de natureza fonológica englobam as "Omissões" (faltam letras na palavra em razão de uma análise fonológica imprecisa ou desconhecimento de correspondências fonemas-grafemas), "Outras" (que correspondem a substituições 
Tabela 10 - Descritiva dos erros para o problema Desconhecido

\begin{tabular}{lccccccccc}
\hline Desconhecido & Média & Mediana & $\begin{array}{c}\text { Desvio } \\
\text { Padrão }\end{array}$ & CV & Q1 & Q3 & N & IC & p-valor \\
\hline representações & 15,25 & 16,5 & 8,45 & $55 \%$ & 9,5 & 22,5 & 8 & 5,85 & \\
múltiplas & 9,00 & 9 & 6,00 & $67 \%$ & 5 & 13,3 & 8 & 4,16 & \\
oralidade & 10,75 & 5,5 & 13,47 & $125 \%$ & 1 & 13,8 & 8 & 9,33 & \\
omissão & 1,13 & 0 & 1,64 & $146 \%$ & 0 & 2,25 & 8 & 1,14 & \\
junção/separação & 1,50 & 1 & 1,77 & $118 \%$ & 0 & 2,25 & 8 & 1,23 & \\
am / ão & 1,25 & 1 & 0,46 & $37 \%$ & 1 & 1,25 & 8 & 0,32 & $<0,001^{*}$ \\
generalização & 2,13 & 2 & 1,96 & $92 \%$ & 0,75 & 3 & 8 & 1,36 & \\
surda/sonora & 2,38 & 2 & 1,77 & $74 \%$ & 1 & 4 & 8 & 1,22 & \\
acréscimo & 1,13 & 0,5 & 1,73 & $154 \%$ & 0 & 1,25 & 8 & 1,20 & \\
letras parecidas & 0,63 & 0,5 & 0,74 & $119 \%$ & 0 & 1 & 8 & 0,52 & \\
inversões & 7,63 & 3 & 9,40 & $123 \%$ & 0 & 15,3 & 8 & 6,51 & \\
outras & & & & & & & & &
\end{tabular}

Tabela 11 - P-valores das comparações entre tipos de erros em Desconhecido

\begin{tabular}{|c|c|c|c|c|c|c|c|c|c|c|}
\hline Desconhecido & $\begin{array}{c}\text { representações } \\
\text { múltiplas }\end{array}$ & oralidade & omissão & junção/sep. & am / ão & general. & sur/son. & acréscimo & letras par. & inversões \\
\hline oralidade & 0,017 & & & & & & & & & \\
\hline omissão & 0,182 & 0,893 & & & & & & & & \\
\hline junção/sep. & 0,012 & 0,012 & 0,011 & & & & & & & \\
\hline am / ão & 0,012 & 0,012 & 0,089 & 0,609 & & & & & & \\
\hline general. & 0,012 & 0,018 & 0,046 & 0,660 & 0,680 & & & & & \\
\hline sur/son. & 0,011 & 0,035 & 0,058 & 0,131 & 0,725 & 0,319 & & & & \\
\hline acréscimo & 0,012 & 0,018 & 0,049 & 0,131 & 0,388 & 0,167 & 0,732 & & & \\
\hline letras par. & 0,012 & 0,018 & 0,065 & 0,785 & 0,461 & 0,666 & 0,246 & 0,244 & & \\
\hline inversões & 0,012 & 0,018 & 0,018 & 0,398 & 0,262 & 0,129 & 0,071 & 0,034 & 0,726 & \\
\hline outras & 0,018 & 0,440 & 0,172 & 0,043 & 0,225 & 0,179 & 0,114 & 0,176 & 0,144 & 0,089 \\
\hline
\end{tabular}

atípicas de letras, por falhas na correspondência fonema/grafema como na troca de "chuva" por "suva"), "Surdas/sonoras" (envolve procedimentos de diferenciação de fonemas pelo traço de sonoridade), "Acréscimos" e "Inversões" (quando se trata de mudar a posição de uma letra dentro da palavra: "carta" escrita como "crata").

\section{Grupo TDA/H}

A maior ocorrência de erros por "Representações Múltiplas", que tende a apresentar diferença significante em relação aos demais tipos de erros, revela que a maior dificuldade encontrada por este grupo diz respeito principalmente a questões de ordem ortográfica, ligadas à possibilidade de um mesmo som poder ser escrito por várias letras e uma mesma letra poder escrever mais do que um fonema. As trocas entre letras correspondentes (por exemplo, x/ch; s/ss/ç), caracterizam estes erros. A hipótese de um déficit ortográfico fica reforçada pela presença também frequente de erros tipo "Oralidade", que são produzidos por uma não compreensão de que escrever não se limita a uma transcrição fonética, envolvendo também regras ortográficas (Por exemplo, embora pronuncie "papel" com /u/ no final - [papeu], para escrever é necessário usar o "l", uma vez que "é" é uma vogal aberta). Este perfil, com uma frequência baixa de omissões, indica que o grupo, como um todo, já atingiu um nível alfabético de escrita restando predominantemente questões de ordem ortográfica, mais complexas.

Os erros envolvendo "Omissões", "Outras alterações" e "Trocas surdas-sonoras", indicam dificuldades ainda não superadas ao nível fonológico, principalmente em relação às correspondências fonemas-grafemas, porém em segundo lugar. As "Inversões" e "Letras parecidas", pela frequência bastante baixa, sugerem ausência de dificuldades no nível visuo-espacial.

\section{Grupo Dificuldades Escolares}

Este grupo que engloba crianças cujas limitações podem ser atribuídas a causas externas, 
como condições não favoráveis de ensino apresenta, como dificuldades principais, tanto erros de natureza ortográfica (Representações Múltiplas), quanto fonológicas (Omissão de letras), uma vez que não há diferença significante entre os dois tipos de erros mais frequentes. A média de erros por omissão pode indicar que este grupo ainda não atingiu um nível alfabético consistente. Os erros por "Oralidade" aparecem em segundo lugar e também dependem de questões ortográficas. Por outro lado, a diferença entre "Oralidade" e "Outros" não é significante, reforçando a idéia de dificuldades no plano ortográfico e fonológico, simultaneamente. Os erros envolvendo aspectos de natureza visuo-espacial têm rara ocorrência.

\section{Grupo distúrbio de aprendizagem}

Os sujeitos deste grupo apresentam dificuldades evidentes em aspectos ortográficos, de acordo com a média de "Representações Múltiplas". Porém, se forem considerados em conjunto, os erros por "Omissão", "Outras" e "Surdas/Sonoras", pode-se afirmar que dificuldades de natureza fonológica estão bastante presentes, inclusive pela média mais elevada de trocas "Surdas/sonoras". A não diferença estatística entre os erros, a partir de "Surdas/sonoras" até "Generalização" parece indicar uma dificuldade mais difusa para estes sujeitos, o que não confirma a hipótese de que nestes casos de distúrbios de aprendizagem, em razão de um déficit mais generalizado de linguagem, teriam um problema maior em aspectos ortográficos ${ }^{22}$. Pode-se supor que a presença de um déficit mais generalizado de linguagem pode produzir uma configuração também mais difusa de erros, como os aqui observados. No terceiro bloco de erros, de baixa frequência, estão os de natureza visuo-espacial.

\section{Grupo Dislexia}

Somente três sujeitos compõem este grupo, o que dificulta uma análise estatística mais pormenorizada. Porém, considerando-se as médias, "Representações múltiplas" e "Oralidade" vêm em primeiro e segundo lugar, respectivamente, ambas indicando que os erros mais frequentemente observados derivam principalmente de questões de ordem ortográfica. Um segundo bloco de erros, constituído por "Omissões", "Outras" e "Surdas/sonoras", indica a presença também de falhas fonológicas e que o nível alfabético não parece ainda bem consolidado. Chama a atenção a média mais elevada de erros do tipo "Surdas/sonoras", os quais confirmam dificuldade no nível fonológico. A ideia de que os erros de escrita na dislexia seriam predominantemente de natureza fonológica ${ }^{22}$ não se confirma com os resultados obtidos com esta amostra, se bem que deve ser considerado que a mesma pode não ser representativa, por seu reduzido tamanho. Também fica evidente que, este grupo, ao contrário do que se afirma no caso de disléxicos, não apresenta dificuldades quanto a aspectos visuo-espaciais, considerando-se a presença mínima de erros do tipo "Letras parecidas" e "Inversões".

\section{Grupo Distúrbios Associados}

"Representações Múltiplas" e "Omissões" formam um primeiro bloco de erros neste tipo de problema, sem que haja uma diferença significante entre estes erros, embora a média de "Representações múltiplas" seja maior. Este dado indica a presença de dificuldades ortográficas e fonológicas ao mesmo tempo. O segundo bloco de erros, também sem diferença significante entre os mesmos, reforça a hipótese de dificuldades simultâneas em aspectos ortográficos ("Oralidade" e "Am / ão") e fonológicos ("Outras", "Surdas/sonoras" e "Acréscimo"). Erros envolvendo aspectos visuo-espaciais não constituem problemas para este grupo.

\section{Grupo com diagnóstico "Desconhecido"}

Este grupo de sujeitos, com diagnóstico em aberto, revela dificuldades maiores principalmente em "Representações múltiplas" que, apesar da média maior, não apresenta diferença significante em relação às "Omissões". O segundo grupamento de erros, por proximidade de frequência, diz respeito à "Oralidade" e "Outras", que coloca em evidência, juntamente com o primeiro grupo, as dificuldades de natureza ortográfica e fonológica. Os demais erros têm uma frequência muito baixa, com as inversões vindo em último lugar.

\section{CONCLUSÕES}

Em todos os grupos, o erro mais frequente é "Representações Múltiplas" indicando que, independentemente do tipo de problema, compreender e dominar as regras ortográficas é uma tarefa complexa. "Oralidade", que no geral é o terceiro tipo de erro mais encontrado, também envolve aspectos relativos à ortografia.

"Omissões" vêm em segundo lugar e, assim como "Outras alterações" envolve questões de análise fonológica. indicando falhas em processos elementares de correspondência fonemagrafema, ainda não superadas. Em crianças sem dificuldades, observa-se que os erros do tipo "Oralidade" têm uma média maior do que as "Omissões". A inversão das frequências pode ser um dado indicativo de problemas no aprendizado da escrita. 
As trocas "Surdas/sonoras" tendem a ter uma média mais aumentada nos grupos "Dislexia", "Distúrbios de aprendizagem" e "Associados", o que pode ser indicativo de uma dificuldade fonológica mais acentuada nestes grupos.

Em nenhum dos tipos de problemas aqui analisados constatou-se dificuldades para escrever invertendo letras ou confundindo letras que podem ser semelhantes, o que significa que as habilidades necessárias para discriminar visualmente as letras distinguindo-as, assim como as habilidades para detectar que posição as letras devem ocupar nas palavras estavam presentes nos sujeitos avaliados.

Há necessidade de um grupo maior de sujeitos para análises mais pormenorizadas, principalmente do grupo "Dislexia". Deve-se também proceder a uma análise para verificar se há diferenças significantes entre os grupos, complementando este trabalho cujo enfoque foi o de uma análise intragrupal.

\section{ABSTRACT}

Purpose: to describe the orthographic findings in several types of learning problems, check if the types of produced mistakes are those found in the learning that is considered normal and analyze if orthographic or phonological nature problems prevail in each disorder. Methods: the writing of 64 subjects was evaluated by the Laboratory of Learning Disabilities of the Neurology Department of UNICAMP and diagnosed as showing some type of learning problem. Deficit of Attention / Hyperactivity disorder (28); School Difficulties (13); Learning Disabilities (7); Dyslexia (3); Associated Disorders (5) and Inconclusive Diagnosis (9). The ages varied between 8;2 and 13;4 years, with a 10;6 yearold average. Only subjects in alphabetical writing level without any type of intellectual deficit were included. The found mistakes were classified in eleven categories and quantified for ends of statistical analysis. Results: the spelling mistakes found in each problem type correspond to those observed in children without learning complaint. The spelling mistakes through Multiple Representations, Omission of letters and Orality, are respectively, the three most frequent types in the cases Deficit of Attention and Hyperactivity disorder, School Difficulties, Associated Disorders and Unknown Diagnosis. In the Disturbance of Learning the sequence is of Multiple Representations, Omission, Other Mistakes and Voiced/Unvoiced mistakes. In the dyslexia we note the sequence of Multiple Representations, Orality, Omission and Other Mistakes. There is a trend, in each problem type, to the prevalence of orthographic nature mistakes, although with no statistically significant difference in relation to the phonological nature mistakes. Conclusion: the orthographic nature mistakes are the most frequent, although, there is no significant difference, in each group, in relation to the phonological nature mistakes. With contrary trend, the visual-spatial mistakes have low occurrence in general, which shows that the difficulty concerning all groups has fundamentally a linguistic origin and not a perceptual.

KEYWORDS: Learning Disorders; Handwriting; Education

\section{REFERÊNCIAS}

1. Caravolas M, Volín J. Phonological spelling errors among dyslexic children learning a transparent orthography: the case of Czech. Dyslexia. 2001; 7(4):229-45.

2. Romonath R, Wahn C, Gregg N. [Phonological and orthographic processes of reading and spelling in young adolescents and adults with and without dyslexia in German and English: impact on foreign language learning]. Folia Phoniatr Logop. 2005; 57(2):96-110.

3. Juul $\mathrm{H}$, Sigurdsson B. Orthography as a handicap? A direct comparison of spelling acquisition in Danish and Icelandic. Scand J Psychol. 2005; 46(3):263-72.
4. Ziegler JC, Goswami U. Reading acquisition, developmental dyslexia, and skilled reading across languages: a psycholinguistic grain size theory. Psychol Bull. 2005; 131(1):3-29.

5. Seymour PHK, Aro M, Erskine JM. Foundation literacy acquisition in European orthographies. $\mathrm{Br}$ J Psychol. 2003; 94(2):143-74.

6. Zorzi JL. Aprender a escrever: a apropriação do sistema ortográfico. Porto Alegre: ArtMed; 1998.

7. Guimarães GE, Roazzi A. A importância do significado na aquisição da escrita ortográfica. In: Morais AG, organizador. O aprendizado da ortografia. Belo Horizonte: Autêntica; 2000.p. 61-76. 8. Morais AG. Ortografia: este espetacular objeto de conhecimento. In: Morais AG, organizador. 
O aprendizado da ortografia. Belo Horizonte: Autêntica; 2000.p. 7-19.

9. Rego LLB, Buarque LL. Algumas fontes de dificuldade na aprendizagem de regras ortográficas. In: Morais AG, organizador. O aprendizado da ortografia. Belo Horizonte: Autêntica; 2000.p. 21-41. 10. Scliar-Cabral L. Princípios do sistema alfabético do português do Brasil. São Paulo: Contexto; 2003. 11. Zorzi JL. Aprendizagem e distúrbios da linguagem escrita. Porto Alegre: ArtMed; 2003.

12. Zanella MSZ. Leitura e aprendizagem da ortografia: um estudo com alunos de $4^{\mathrm{a}}$ a $6^{\mathrm{a}}$ série do Ensino Fundamental [tese]. São Paulo (SP): Pontifícia Universidade Católica; 2007.

13. Grigalevicius MM. Aprendizagem da linguagem escrita: um estudo sobre a competência ortográfica de alunos da $5^{\text {a }}$ série do ensino fundamental [dissertação]. São Paulo (SP): Pontifícia Universidade Católica; 2007.

14. Santos TMS. Vocabulário, consciência fonológica e nomeação rápida: contribuições para a ortografia e elaboração escrita. [tese]. São Paulo (SP): Universidade de São Paulo; 2007.

15. Osmon DC, Braun MM, Plambeck EA. Processing abilities associated with phonologic and orthographic skills in adult learning disability. J Clin Exp Neuropsychol. 2005; 27(5):544-54.

16. Papagno C, Girelli L. Writing through the phonological buffer: a case of progressive writing disorder. Neuropsychol. 2005; 43(9):1277-87.

17. Connelly V, Campbell S, MacLean M, Barnes J. Contribution of lower order skills to the written composition of college students with and without dyslexia. Dev Neuropsychol. 2006; 29(1):175-96.

18. Silliman ER, Bahr RH, Peters ML. Spelling patterns in preadolescents with atypical language skills: phonological, morphological, and orthographic factors. Dev Neuropsychol. 2006; 29(1):93-123.

19. Ciasca SM, Moura-Ribeiro MVL. Avaliação e manejo neuropsicológico da dislexia. In: Rotta NT, Ohlweiler L, Riesgo RS. Transtornos da aprendizagem: abordagem neurobiológica e multidisciplinar. Porto Alegre: Artmed; 2006.p. 181-94.

RECEBIDO EM: 07/02/2009

ACEITO EM: 27/07/2009

Endereço para correspondência:

Jaime Luiz Zorzi

Rua Cayowaá, 664

São Paulo - SP

CEP: 05018-000

E-mail: jaime@ cefac.br
20. Zorzi JL. Os distúrbios de aprendizagem e os distúrbios específicos de leitura e da escrita. In: Britto ATBO, organizador. Livro de fonoaudiologia. São José dos Campos: Pulso Editorial; 2005. p. 217-30.

21. Zorzi JL. Alterações ortográficas nos transtornos de aprendizagem. In: Maluf MI, organizador. Tramas do conhecimento, do saber e da subjetividade. Petrópolis: ABPp / Editora Vozes; 2006.p. 144-62.

22. Gustafson S, Ferreira J, Rönnberg J. Phonological or orthographic training for children with phonological or orthographic decoding deficits. Dyslexia. 2007; 13(3):211-29.

23. Goswami U. Phonological representations, reading development and dyslexia: towards a crosslinguistic theoretical framework. Dyslexia. 2000; 6(2):133-15.

24. Spencer K. Is English a dyslexic language? Dyslexia. 2000; 6(12):152-62.

25. Spencer K. Differential effects of orthographic transparency on dyslexia: word reading difficulty for common English words. Dyslexia. 2001; 7(4):217-28.

26. Capovilla AGS, Joly MCRA, Ferracini F, Caparrotti NB, Carvalho MR, Raad AJ. Estratégias de leitura e desempenho em escrita no início da alfabetização. Psicol Escol Educ. 2004; 8(2):189-97. 27. Meireles ES, Correa J. Regras contextuais e morfossintáticas na aquisição da ortografia da língua portuguesa. Psicol Teor Pesq. 2005; 21(1):77-84.

28. Ciasca SM. Avaliação neuropsicológica e neuroimagem nos distúrbios de aprendizagem, leitura e escrita. In: Associação Brasileira de Dislexia. Dislexia: cérebro, cognição e aprendizagem. São Paulo: Frontis; 2000.

29. Shaywitz SE, Shaywitz BA. Dyslexia (specific reading disability). Biol Psychiatr. 2005; 57(11):1301-9.

30. Catts HW. Phonological awareness: putting research into practice. Lang Learn Educ. 1999; 6(1):17-9.

31. Griffiths S, Frith U. Evidence for an articulatory awareness deficit in adult dyslexics. Dyslexia. 2002; 8(1):14-21.

32. Bourassa D, Treiman R. Spelling in children with dyslexia: analyses from the Treiman-Bourassa early spelling test. Sci Stud Read. 2003; 7(4):309-33.

33. Cao F, Bitan T, Chou TL, Burman DD, Booth JR. Deficient orthographic and phonological representations in children with dyslexia revealed by brain activation patterns. J Child Psychol Psychiatr. 2006; 47(10):1041-50.

34. Mackie C, Dockrell JE. The nature of written language deficits in children with SLI. J Speech Lang Hear Res. 2004; 47(6):1469-83. 